\title{
Los Principios de Florencia sobre el doctorado en ARTes*
}

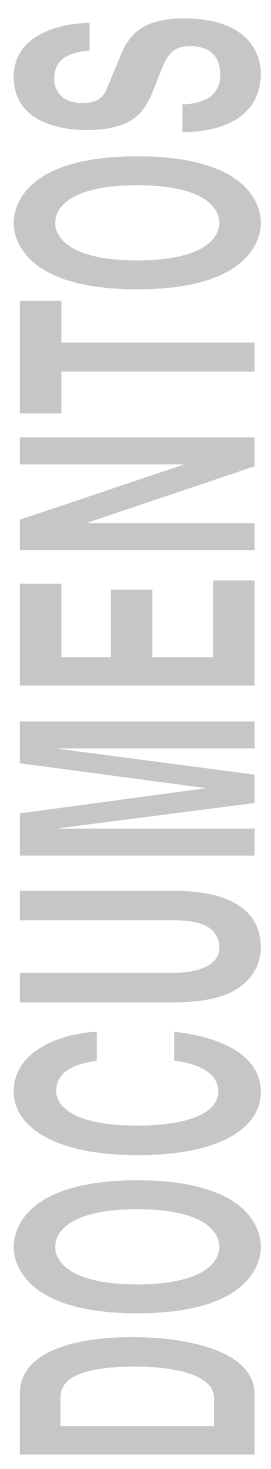

\section{CON EL PATROCINIO Y APOYO DE}

AEC Asociación Europea de Conservatorios

CILECT Asociación Internacional de Escuelas de Cine y Televisión

(Centre International de Liaison des Écoles de Cinéma et de Télévision, CILECT)

Cumulus Asociación Internacional de Universidades y Escuelas de Arte, Diseño y Medios EAAE Asociación Europea para la Enseñanza de la Arquitectura

SAR Sociedad de Investigación Artística

\section{Prólogo}

El presente trabajo pretende ser un documento expositivo sobre el doctorado en artes ${ }^{1}$. Está formulado como punto de referencia para legisladores, dirigentes universitarios, diseñadores de planes de estudios y organismos de financiación para la investigación. Asimismo, está dirigido a universidades de artes y ciencias con el fin de ayudar a las primeras a obtener reconocimiento para sus proyectos (ante organismos de financiación nacionales, legisladores, etc.) y a las segundas a conocer los avances de la investigación en el ámbito de las universidades de artes. Este trabajo nace de la inclusión de la investigación artística en el Manual de Frascati de la OCDE y tiene la intención de seguir dando forma al conocimiento de la investigación en el campo de las artes y de crear los marcos de trabajo, los entornos y los recursos necesarios para que los investigadores en fase inicial (investigadores doctorales) desarrollen sus proyectos. Por último, en este trabajo se señala que todo lo que es válido para la investigación doctoral y la creación de estudios de doctorado -en los términos definidos en documentos fundamentales

*Traducción del siguiente texto original: The Florence Principles' on the Doctorate in Arts (2016). Trabajo desarrollado por el Grupo de Trabajo de Investigación Artística creado por la Liga Europea de Institutos de las Artes (ELIA) y presentado en la Conferencia Bienal de ELIA, celebrada en Florencia el 2 de diciembre de 2016, acceso 20 de noviembre de 2020, https://cdn.ymaws.com/eliaartschools.org/resource/resmgr/newfolder/26-september-florence-princi.pdf. Traducción realizada por el Servicio de Traducción de la Universidad de Alcalá.

${ }^{1}$ Para simplificar, el término «doctorado en artes» se utiliza en este trabajo para describir todos los formatos de programas de tercer ciclo en arte. Asimismo, este término hace referencia a todas las disciplinas y áreas artísticas (es decir, bellas artes, música, drama, artes escénicas, arquitectura, etc.). 
como Salz̧burg Recommendations (2005) y Taking Salzburg Forward (2016), ambos de la Asociación Europea de Universidades, o EUA por su denominación en inglés, o Principles for Innovative Doctoral Training (de la Comisión Europea) en el marco de la UE- también se aplica a los estudios de doctorado en las artes. Por muy diferentes que puedan parecer los resultados de investigaciones, los procesos, la motivación epistemológica y la coherencia con la que se llevan a cabo los proyectos de investigación en el ámbito de las artes siguen siendo los mismos.

Este trabajo ha sido desarrollado por el Grupo de Trabajo de Investigación Artística creado por la Liga Europea de Institutos de las Artes (ELIA por su denominación en inglés), que también incluye a representantes de la Sociedad de Investigación Artística (SAR), la Asociación Europea de Conservatorios (AEC) y la Asociación Europea para la Enseñanza de la Arquitectura (EAAE). Su borrador fue redactado por miembros de este grupo ${ }^{2}$, tratado con un grupo de trabajo del Consejo de Educación Doctoral de la $\mathrm{EUA}^{3}$ y editado por el grupo de trabajo de ELIA hasta la versión final. El trabajo recibió la aprobación del consejo directivo de ELIA el 4 de noviembre de 2016 y se presentó en la Conferencia Bienal de ELIA, celebrada en Florencia el 2 de diciembre del mismo año.

\section{Parte A: Contexto}

Aproximadamente 280 instituciones de todo el mundo ofrecen títulos de investigación en las artes (bellas artes, diseño, música, arquitectura, danza, teatro y todas las demás disciplinas artísticas). La estructura administrativa de las instituciones que conceden dichos títulos varía mucho, al igual que el nombre de los títulos que ofrecen (doctor en Artes Creativas [DCA], doctor en Humanidades [DPhil/ $\mathrm{PhD}$ ] o doctor en Bellas Artes [DFA]). Todas estas instituciones tienen puntos fuertes específicos y presentan diferencias en la evaluación, la financiación, los niveles de alumnos internacionales y, por supuesto, en el cuerpo docente y no docente. Sin embargo, comparten el mismo interés: hacer realidad programas de doctorado que permitan a los artistas «desarrollar conocimientos a través de la investigación original (usando metodologías artísticas y de otras disciplinas)» ${ }^{4}$.

Durante las últimas cuatro décadas, el doctorado en artes se ha asentado adoptando diversos títulos y formas a lo largo y ancho de la UE, así como en otras regiones. Por ejemplo, algunos países (Reino Unido, Noruega, Suecia y España) llevan diez años o más concediendo títulos de doctorado en artes y, aunque otros han comenzado a introducir estudios de tercer ciclo en la última década, algunos no han decidido hacerlo hasta hace poco.

${ }^{2}$ Andrea B. Braidt (presidenta), Giaco Schiesser (copresidente, SAR), Cecile Broch-Knudsen, Anna Daucikova, Peter Dejans (AEC), Lars Ebert (oficina de ELIA), Henry Rogers y Johan Verbeke (EAAE).

${ }^{3}$ En un taller celebrado en Zúrich en mayo de 2016; Melita Kovacevic, Edwin Constable y Thomas Jorgensen como miembros de la EUA.

${ }^{4}$ Salz̧burg Principles on Doctoral Education, publicación de la EUA, 2005. 
Dado que las condiciones jurídicas de los marcos nacionales varían con relación a la introducción de estudios de investigación doctoral en artes o en diseño, podemos hablar en este sentido de una Europa de varias velocidades. Por ejemplo, mientras que ya en 2003 la legislación noruega permitía la introducción de un diploma de tercer ciclo en investigación artística, la Ley de Universidades de Austria no se enmendó hasta 2015 para incluirlo.

Las universidades de artes también varían con respecto a su estatus institucional. Muchos países tienen universidades de artes autónomas con el mismo estatus (y las mismas normas legislativas) que las denominadas universidades «generales» (p. ej., Austria o Suecia); otros países organizan las universidades de artes del mismo modo que las universidades de ciencias aplicadas (como ocurre en Suiza, con las Fachbochschulen, o en los Países Bajos), o bien como academias (p. ej., Italia) o como facultades que forman parte de universidades «clásicas» (p. ej., España o Croacia). En consecuencia, la puesta en marcha de programas de doctorado en las artes está sujeta a diversos procesos y disposiciones reglamentarias. Mientras que algunos países han introducido doctorados en las artes como parte de programas de estudio de cooperación con universidades científicas (p. ej., en Suiza y en partes de Bélgica), las universidades de artes de otros países han podido poner en marcha doctorados artísticos por derecho propio, sin necesidad de cooperación (p. ej., Reino Unido, Noruega, Finlandia, Austria o República Checa). Con independencia de las diferencias estructurales que pueda haber, las discusiones en torno al doctorado en artes -lideradas por ELIA, universidades de artes y otras organizacioneshan demostrado que existe una creciente motivación para ofrecer programas de doctorado por toda Europa y en otras regiones.

Esta creciente motivación coincide con el enorme desarrollo de la investigación artística en todas las disciplinas relacionadas y, hasta cierto punto, es consecuencia de este. Al mismo tiempo, existe una comunidad internacional de investigación artística, un grupo de ámbito internacional y europeo de organizaciones de investigación artística (p. ej., la Red Europea de Investigación artística [EARN por su denominación en inglés], la Sociedad de Investigación Artística [SAR], la Plataforma Europea de Investigación Artística en Música [EPARM]), multitud de organizaciones nacionales (p. ej., la Plataforma para la Investigación Artística de Suecia [PARSE]), varias revistas arbitradas dedicadas a la difusión de resultados de investigaciones artísticas (p. ej., el Journal for Artistic Research [JAR] o la revista de la PARSE) y gran cantidad de programas de financiación para proyectos de investigación artística (p. ej., el programa PEEK del Fondo Científico de Austria, el Programa de Investigación Artística de Noruega y el programa de financiación para investigación artística del Consejo de Investigaciones de Suecia). Abordar preguntas de investigación con métodos artísticos y crear obras de arte que asuman los grandes retos de investigación y desarrollo que plantea Europa -y, por supuesto, el mundo entero- se ha convertido en un cometido dinámico e innovador. En 2015, la OCDE respondió incluyendo la investigación artística como clasificador de la investigación y el desarrollo en el Manual de Frascati, con lo que preparó el terreno 
de una mayor inclusión en los marcos de investigación europeos 5 . Por ejemplo, el Consejo Europeo de Investigación ha incorporado la investigación artística en sus regímenes de financiación.

Si analizamos la evolución de la investigación artística y de los doctorados en las artes durante los últimos 20 años, resulta evidente que ha tenido lugar un debate global y que han comenzado a desarrollarse estándares comunes de dichos doctorados.

Se ha identificado una serie de temas comunes, que se han convertido en objeto de discusión en el seno de la comunidad de investigación artística y de las universidades de artes, tanto a nivel nacional como europeo. Así, se ha encontrado una generalidad fundamental en dicha comunidad: un doctorado en artes cumple los requisitos previos para la obtención de un doctorado en Humanidades, en los términos en que se formulan en las ciencias y las humanidades y se describen, por ejemplo, en documentos expositivos europeos, como Salzburg Recommendations (de la EUA) o Principles of Innovative Doctoral Training (de la Comisión Europea).

Los debates internacionales sobre los doctorados en artes pueden agruparse en torno a dos ejes. Por un lado, la discusión se ha centrado en cuestiones institucionales de orden práctico relacionadas con las normativas de los títulos de doctorado (p. ej., la admisión, la evaluación, los requisitos de la tesis, los cursos impartidos y la medida en que debería ser obligatorio) y la financiación de los doctorandos (empleo, becas, etc.). Por otro lado, se han identificado cuestiones sobre lo que hay en juego con relación a una serie de áreas estratégicas, entre ellas ${ }^{6}$ :

- La Declaración de Bolonia -en la que se ubicó el doctorado en Humanidades- y sus efectos.

- Los formatos para presentar los resultados de doctorados en Humanidades y la importancia del discurso inherente.

- La discusión en torno a las mejor prácticas ${ }^{7}$ y a sus referentes, sin fijar un canon normativo ni enredarse en descripciones poco precisas de criterios.

- La función, naturaleza y formación de los directores de tesis ${ }^{8}$.

- La organización y estructura de los programas de doctorado en artes (p. ej., doctorados en Humanidades individuales, grupos de investigación o escuelas de estudios superiores mixtas).

${ }^{5}$ OCDE, Medición de las actividades cientificas, tecnológicas y de innovación. Manual de Frascati 2015.

${ }^{6}$ P. ej.: Schiesser, G. (2015). What is at stake - Qu'est ce que l'enjeu? Paradoxes - Problematics - Perspectives in Artistic Research Today. En G. Bast y E.G. Carayannis (eds.), Arts, Research, Innovation and Society [= ARIS, vol. 1] (Nueva York: Springer).

${ }^{7}$ Puede consultarse una recopilación de 15 casos prácticos, incluidos comentarios, en: Wilson, M. y van Ruiten, S. (eds.). SHARE Handbook for Artistic Research Education, nota al pie 2, pp. 74-120.

${ }^{8}$ P. ej.: 3. ${ }^{a}$ conferencia del EUFRAD (celebrada en Viena en septiembre de 2013) sobre «la experiencia y los conocimientos de los directores en el desarrollo y realización de trabajo doctoral en el ámbito de las artes». 
- Sostenibilidad: ¿qué formatos adecuados para la difusión de resultados de trabajo doctoral en las artes deberían desarrollarse con urgencia??

- Empleabilidad y diferentes perspectivas profesionales de los doctores en Humanidades: ¿contribuyen los programas de doctorado en las artes principalmente al desarrollo de la persona como artista? ¿De qué formas se integra a los doctores en Humanidades en el entorno de investigación de las universidades de artes?

- Internacionalización.

En la siguiente sección «Parte B: Relaciones»- se hace referencia a documentos expositivos que resultan pertinentes para nuestra discusión. A continuación, en la sección C del presente trabajo, nos centramos en siete puntos que podrían servir de pilares de orientación en la discusión. Estos puntos, de retórica descriptiva más que normativa, llaman la atención sobre los aspectos importantes para el desarrollo y posterior éxitos de doctorados en artes.

\section{Parte B: Relaciones}

En esta sección se identifican los documentos normativos y estratégicos que han fundamentado el discurso en torno a los doctorados en artes. Estos documentos han dado forma a nuestra discusión y, en distintos grados, constituyen la esencia y base de nuestra concepción de los «puntos de atención» que se exponen en la siguiente sección.

La publicación de Salz̧burg Principles (Principios de Salzburgo) ${ }^{10}$ en 2005 sentó las bases para abordar la educación doctoral como parte del proceso de Bolonia. En este documento se identificaron diez principios para el desarrollo de títulos de tercer ciclo: la formación doctoral como desarrollo del conocimiento a través de la investigación original; la incorporación de la formación doctoral en las estrategias y políticas institucionales; la importancia de la diversidad; la consideración de los doctorandos como investigadores en fase inicial; el papel crucial de la supervisión y la evaluación; la consecución de una masa crítica como objetivo importante de los programas de doctorado; la necesidad de que

${ }^{9}$ La revista arbitrada Journal for Artistic Research (JAR) ofrece un enfoque prometedor, al permitir simultáneamente investigación artística propiamente dicha, documentación, evaluación externa por expertos y formatos híbridos de difusión de resultados de doctorados en Humanidades.

${ }^{10}$ Conclusiones y recomendaciones del seminario de Bolonia «Doctoral Programmes for the European Knowledge Society» (Programas de doctorado para la sociedad europea del conocimiento), celebrado en Salzburgo del 3 al 5 de febrero de 2005. Los principios son el resultado del seminario de Salzburgo, organizado por el Ministerio de Educación, Ciencia y Cultura de Austria, el Ministerio de Educación e Investigación de Alemania y la Asociación Europea de Universidades (EUA por su denominación en inglés). El propósito y objetivo principal del seminario era identificar los retos claves que deberían abordarse a la hora de poner en práctica la Línea de actuación (durante el período 2005-2007). 
el estudio dure de tres a cuatro años; la promoción de estructuras innovadoras; la importancia de la movilidad para los investigadores doctorales; y una financiación adecuada de los doctorandos como requisito previo. Estos principios, formulados como intenciones hace más de diez años, siguen siendo parte integrante de casi todas las discusiones sobre educación doctoral, aunque muchos de ellos se han convertido en certezas desde entonces.

En 2010, la EUA publicó Sal̨̧burg II Recommendations (Recomendaciones de Salzburgo II) ${ }^{11}$, un trabajo que, a partir de los diez principios, pretendía servir de documento de referencia para quienes estuvieran dando forma a la educación doctoral en su país o institución, o bien participaran en otros aspectos del proceso de reforma de la educación doctoral ${ }^{12}$. Las recomendaciones planteaban cambiar las estructuras universitarias, hacer hincapié en el papel de la institución en el proceso doctoral y desterrar el modelo tradicional de supervisión individualizada.

En 2011, la Dirección General de Investigación y Desarrollo de la Comisión Europea publicó un trabajo que influiría en la discusión sobre educación doctoral y se traduciría en el documento expositivo Principles for Innovative Doctoral Training (Principios de una formación doctoral innovadora) ${ }^{13}$. Basándose en Salz̧burg Principles, el documento de la Comisión añadía la formación en competencias transferibles y el control de calidad a la lista de recomendaciones aplicables a la educación de tercer ciclo. Asimismo, se añadió un punto sobre la «exposición [de los doctorandos] a la industria y a otros sectores de empleo pertinentes», que recogía la noción de «empleabilidad» del proceso de Bolonia, una noción muy discutida (y criticada).

Todos los documentos anteriormente mencionados -además, por supuesto, del recientemente publicado Taking Sal̨̧burg Forward (de la EUA) ${ }^{14}$, que añade las dimensiones de ética investigadora, orientación global y la necesidad de interactuar con partes interesadas no pertenecientes al ámbito académico- proporcionan puntos de referencia cruciales para el marco de los doctorados en artes o en diseño.

En los últimos cinco años, hemos asistido a la publicación de varios documentos expositivos, libros blancos, etc., surgidos del ámbito de las artes, acerca de la investigación artística y, por ende, los estudios doctorales. Por ejemplo, la Asociación Europea para la Enseñanza de la Arquitectura (EAAE por su denominación en inglés) aprobó en 2013 su Charter for Architectural Research, una carta en la que recalcaba la necesidad de canales específicos y globales de comunicación del conocimiento en el

${ }^{11}$ Salz̧burg II Recommendations. European Universities' Achievements since 2005 in Implementing the Salzburg Principles. EUA, 2010.

${ }^{12}$ Kovacevic, M. y Mihaljevic, S. (2016). New Developments in Doctoral Education. En L. Zinner (ed.), Professionals in Doctoral Education (1-14. 5). Viena.

${ }^{13}$ Principles for innovative Doctoral Training, Comisión Europea, Dirección General de Investigación y Desarrollo, Dirección B Espacio Europeo de Investigación, Unidad B.2 «Competencias».

${ }^{14}$ Taking Salzburg Forward. New EUA-CDE Recommendations on doctoral education (2016). 
ámbito de la investigación, que abarcaran proyectos artísticos y académicos ${ }^{15}$. Asimismo, la Asociación Europea de Conservatorios (AEC) publicó un libro blanco en el que reivindicaba el importante papel que juega la investigación artística en el ámbito de las artes musicales. En dicho libro blanco se afirma que la «investigación artística debe aspirar a los mismos estándares de procedimiento que se aplican en todo el espectro de la investigación -replicabilidad (especialmente de procedimientos), verificabilidad, justificación de las afirmaciones citando pruebas, etc.-, aunque, especialmente en áreas como la replicabilidad, debe gozar de libertad para alcanzar estos estándares de formas específicas de su propia naturaleza y de la naturaleza individual y subjetiva de la práctica artística» ${ }^{16}$.

La publicación más completa sobre los estudios de tercer ciclo y la investigación artística corrió a cargo de ELIA, como parte del proyecto SHARE (siglas en inglés de Cambio sustancial para mejorar la investigación y educación en las artes) de 2010-2013, enmarcado en el Programa de Aprendizaje Permanente de la UE. El SHARE Handbook contiene una descripción general del desarrollo de programas doctorales en las artes en Europa e identifica numerosos ejemplos de prácticas recomendadas para tesis y programas doctorales de todo el continente. Este documento aporta una nueva perspectiva al debate, al incorporar a destacados representantes de la comunidad de la investigación artística. Asimismo, proporciona una serie de herramientas para crear planes de estudios a través de reflexiones sobre metodologías empleadas por la investigación en artes y un estudio exhaustivo sobre la cuestión de las (nuevas) disciplinas ${ }^{17}$.

Dado que el principal objetivo (y éxito) del proyecto SHARE fue crear una amplia red de instituciones y personas que invirtieran en educación doctoral en las artes, la publicación final refleja un esfuerzo mayúsculo por reunir una comunidad investigadora europea relevante, recogiendo y reflejando numerosas voces diferentes del ámbito.

Los siete «puntos de atención» de la siguiente sección se basan en todos los trabajos mencionados anteriormente. Dichos puntos intentan extraer el núcleo fundamental de la educación doctoral en las artes y buscan proporcionar pilares de orientación para un campo que lleva desarrollándose durante aproximadamente los últimos 20 años.

\footnotetext{
${ }^{15}$ EAAE Charter on Architectural Research (2012).

${ }^{16}$ Conceptos claves para miembros de la AEC. Investigación artística. Libro blanco del Consejo de la AEC (2015).

${ }^{17}$ M. Wilson y S. van Ruiten (eds., 2013). SHARE Handbook. for Artistic Research Education.
} 


\section{Parte C: Los Principios de Florencia - siete puntos de atención}

\section{- Preámbulo}

Los estudios de doctorado (programas doctorales y $\mathrm{PhD}$ ) en artes permiten a los doctorandos realizar una contribución original a su disciplina. Los programas de estudios de doctorado en artes tienen como objetivo desarrollar la competencia artística, generar nuevos conocimientos y fomentar la investigación artística. Ofrecen a los doctorandos la posibilidad de progresar como artistas e investigadores, al ampliar su competencia artística y su capacidad para crear y compartir nuevas ideas aplicando métodos artísticos innovadores. Los principios generales de la formación doctoral que se formulan en Salzburg Recommendations II y Principles on Innovative Doctoral Training se consideran en buena parte válidos en las artes. En los siguientes puntos se detallan los criterios que son esenciales para los estudios de doctorado en artes, y en cierta medida también específicos de estos.

\section{- Titulación}

Los doctorados en las artes proporcionan una titulación de investigador que se basa en estudios de diploma o máster y exige el desarrollo en profundidad de un proyecto de investigación artística. Los doctorandos se seleccionan con arreglo a los requisitos formales definidos por las instituciones y de acuerdo con su formación y sus competencias académicas. Los posibles directores pueden formar parte del proceso de selección con el fin de garantizar la calidad académica del proceso de elaboración de la tesis.

\section{- Perspectivas profesionales}

Quienes obtengan el título de doctor en artes pueden iniciar (o proseguir) su trayectoria académica en una institución de educación superior o iniciar (o proseguir) su carrera como artistas. Dado que el doctorado en artes suele desarrollarse cuando el doctorando ha completado estudios de posgrado y producido un conjunto considerable de obras, los grupos de los programas de doctorado se componen de artistas asentados con movilidad internacional. Al reunir su experiencia académica y profesional, los grupos crean valiosas redes y acumulan competencias transferibles claves que dan forma a las perspectivas futuras de los doctorandos en las artes. Al finalizar sus estudios, los doctores tienen la posibilidad de compaginar su carrera como artistas con una carrera en la educación superior.

\section{- Trabajo de doctorado}

El trabajo (proyecto de tesis) que se realiza durante los estudios de doctorado en artes incluye el desarrollo de un proyecto de investigación artística original y concreto. En este proyecto se utilizan técnicas y métodos artísticos, por lo que el resultado es una aportación original que genera ideas y conocimientos nuevos en el ámbito artístico. El proyecto consta de obras de arte originales y contiene un componente discursivo en el que se reflexiona de forma crítica sobre el proyecto y se documenta el proceso de investigación. Muchas prácticas artísticas tienen implícito un carácter internacional, interdisciplinario e intercultural, y pueden beneficiarse de los programas de doctorado en artes. 


\section{- Entorno de investigación}

Los estudios de doctorado artísticos que tienen lugar en un entorno de investigación adecuado garantizan el mejor progreso (inter)disciplinario posible para ese trabajo. Un entorno de investigación adecuado consta de una masa crítica de docentes e investigadores doctorales, un perfil activo de investigación artística y una infraestructura eficaz de dimensión internacional (mediante colaboraciones, alianzas, redes, etc.). Los proyectos de investigación doctoral en las artes pueden potenciar disciplinas y un trabajo interdisciplinario, al ampliar las fronteras y entablar nuevas relaciones transversales. Los proyectos de doctorado artísticos exigen una infraestructura y recursos adecuados, en concreto, un estudio y espacios en los que puedan tener lugar exposiciones o actuaciones.

La financiación de los investigadores doctorales en artes es un aspecto crucial.

\section{- Supervisión}

La supervisión es una cuestión fundamental para garantizar buenas prácticas en la educación doctoral; en este sentido, se recomienda que haya al menos dos directores. Un documento de compromiso doctoral, en el que se exponen los roles de supervisión (doctorando-directorinstitución), triangula este proceso y establece los derechos y las obligaciones de todas las partes. Las instituciones instauran una cultura de buena supervisión definiendo de forma precisa las responsabilidades en sus directrices, que sirven de base para evitar y resolver conflictos. La supervisión debe separarse (al menos parcialmente) de la calificación final (evaluación y revisores); por su parte, los directores deben centrarse en mantener la calidad del proyecto de tesis con arreglo a estándares nacionales e internacionales. Los programas de doctorado en artes siguen los procedimientos estándares de control de calidad y valoración aplicables en el contexto nacional e institucional pertinente (acreditación, revisiones, etc.).

\section{- Difusión}

Los resultados del trabajo de doctorado en artes se difunden a través de los canales adecuados. En el caso de trabajo artístico, las exposiciones, las actuaciones, los montajes y el contenido multimedia, los sitios web, etc. proporcionan marcos de difusión adecuados. Debe hacerse especial hincapié en crear instalaciones adecuadas para archivar los resultados de trabajo de doctorado. Siempre que sea posible, y con arreglo a lo dispuesto en el reglamento sobre derechos de autor pertinente, el acceso abierto es el principio rector para difundir el trabajo de investigación artística y la documentación del trabajo artístico (p. ej., carpetas de trabajo digitales en archivos institucionales). Se dará prioridad a contextos con evaluación por pares o validados externamente (p. ej., mediante programas de exposición en museos o procesos de selección comisariados). La singularidad de los contextos de difusión debe explicarse al inicio de los estudios de doctorado (p. ej., en el documento de compromiso doctoral). 


\section{Agradecimiento especial al grupo de trabajo}

Andrea Braidt, Academia de Bellas Artes de Viena

Anna Daucikova, Academia de Bellas Artes de Praga

Peter Dejans, Instituto Orpheus

Cecilie Broch Knudsen, Programa de Investigación Artística de Noruega Henry Rogers, Escuela de Arte de Glasgow

Giaco Schiesser, Universidad de las Artes de Zúrich (ZHdK)

Johan Verbeket, Escuela de Arquitectura de Aarhus y KU Leuven. 